\title{
Analysis of Plant Type and Color Preference and Psychological Assessment for Gardening Activities of Firefighters
}

\author{
Hye Sook Jang ${ }^{1}$, Eunha Yoo ${ }^{1}$, Jeong Hee Kim ${ }^{1 *}$, Sun-Jin Jeong ${ }^{1}$, Jae Soon Kim ${ }^{1}$, and Doo Young Ryu² \\ ${ }^{1}$ Researcher, National Institute of Horticultural \& Herbal Science, RDA, Wanju-gun 55365, Korea \\ ${ }^{2}$ Researcher, Youdeungmeun Damsunro 1548 Agricultural Technology Center Sunchang-gun, Jeollabuk-do 56025, Korea
}

\section{ABSTRACT}

Background and objective: This study investigated plant type and color preference and conducted a psychological assessment for gardening activities of firefighters in a high-risk occupational group.

Methods: A survey was conducted on 117 firefighters aged 39.01 \pm 9.17 in Sunchang in March 2020 to examine their preferences in plant type and color and conduct a psychological assessment of firefighters on gardening activities.

Results: For plant experience and awareness, plant-related event showed the highest score, followed by plant preference tendency and plant growing experience. The level of plant gardening activities was higher among older age and higher job position. As a result of examining the quality of life according to the demographic characteristic with WHOQOL-BREF (World Health Organization Quality of Life Assessment Instrument), it was found that the quality of life was higher among the 20s-30s than 50s, firefighter position than fire lieutenant or higher, those with higher average monthly income, and the singles group than married $(p<.05)$. The administration group showed lower scores than the fire suppression group working in the field, showing statistical significance. Most firefighters showed high preference for herbal plants among the 12 types of flowering plants, in the order of Rosmarinus officinalis > Lavandulaspp. > Mentha piperita, Calendula officinalis. Pelargonium spp. was preferred the least out of the flowering plants, Lycopersicon esculentum was preferred the most out of 12 types of vegetable plants, while Angelica acutiloba was preferred the least statistical significance. For the preference of plant colors, yellow was the most preferred color, followed by white, blue, orange, red, and green, showing statistical significance. For the psychological survey responses of emotion words toward 6 plants colors, it was found that 'bright' was the emotion felt most strongly by the white, yellow, and orange groups, 'calm' and 'comfortable' by the green and blue groups, and 'fancy' by the red group.

Conclusion: The effect of agro-healing programs can be increased by using mostly yellow plants when creating indoor healing gardens at the workplaces of fire officials in a high-risk occupational group, and using herbal plants and Lycopersicor, esculentum in the gardens for agro-healing activities.

Keywords: fancy, fire lieutenant, world health organization quality of life assessment instrument (WHOQOL-BREF)

\section{Introduction}

Frequent exposure to dangerous scenes for fire officials may cause more severe physical and emotional stress than people with other jobs (Whoqol Group, 1995). Symptoms of stress, combined with all kinds of stress caused by poor working conditions, serve as a big obstacle to fire officials in performing their duties beyond just a mental illness (Lee and Choi, 2006). Moreover, social workers who belong to a high-risk occupational group (Sisa IN, 2013, October 17) claim to suffer from severe stress after talking to or intervening with people who experienced a dangerous event

This study was supported by the 2020 Horticultural and Herbal Science Program of the National Institute of Horticultural and Herbal Science of the Rural Development Administration (PJ01514802).

Received: September 14, 2020, Revised: October 6, 2020, Accepted: October 11, 2020

First author: Hye Sook Jang, jhs915@korea.kr, (1) https://orcid.org/0000-0003-3011-8694

*Corresponding author: Jeong Hee Kim, kimjhee@korea.kr, (1D) https://orcid.org/0000-0002-3902-2309 
(Ko, 2007). In particular, stress from the performance of duties puts the person in psychological tension that causes negative behavior, deteriorating the quality of life (Choi, 2001). The World Health Organization (WHO) defined quality of life as "individual's perception of their position in life in the context of the culture and value systems in which they live and in relation to their goals, expectations, standards and concerns" (Whoqol Group, 1995; Lee, 2016). Fire officials may feel mental pressure that an emergency may occur anytime and anywhere, which has a great impact on deteriorating the quality of life (Kang, 2012). Moreover, stress of fire officials may lead to accidents or diseases, result in symptoms such as somatization, obsession, and depression (Kim et al., 1989), and deteriorate the quality of personal life (Oh, 2006). Therefore, a majority of fire officials are classified as risk groups regarding stress, and approximately $17 \%$ of them are high-risk groups (Kim et al., 2007). Post-traumatic stress disorder (PTSD) of fire officials showed a negative correlation with the five sub-factors of the Korean version of WHOQOL-BREF, such as overall quality of life, physical health, and psychological domain, whereas resilience showed a positive correlation with overall quality of life, physical health, and psychological domain, indicating that higher quality of life leads to lower PTSD and higher resilience (Park, 2015; Lee, 2019). High resilience alleviates stress and makes it possible to adapt to stressful situations (Lee et al., 2004; Ko and Yun, 2007; Bak et al., 2010). In particular, natural environment that can improve resilience is the best restorative environment that can relieve fatigue and stress (Kaplan and Kaplan, 1989). Moreover, natural environment or botanical landscape reduces the amount of stress for people who look at it with a sense of stability presented by the green (Ulrich et al., 1991; Parsons et al., 1998), and is highly effective in improving the quality of life (Son et al., 2003). Vision accepts most information (70\%) out of the five human senses (Whang et al., 1997), which is why it is important to understand the visual image of plants (Lee and Bang, 1996). In the visual environment, color is closely correlated with emotions and has an emotional character, affecting human emotions and sensitivity by making them pleased or sad (Kim, 2003). In particular, blue and green are generally known to have high emotional preference (Guilford and Smith, 1959; Valdez and Mehrabian, 1994; Janssens, 2001; Chong et al., 2004; Ou et al., 2004). However, there is insufficient information on colors preferred by firefighters. Moreover, fire officials are classified as high-risk occupational group suffering from PTSD due to frequent exposure to traumatic events, irregular shifts, and high level of job stress due to the nature of their duties (Ryu, et al., 2017), but there are not many studies on actual intervention programs for them. Therefore, it is necessary to improve the mental and physical environment for fire officials in various ways to improve the quality of their lives and reduce stress, such as creating a rest area to relax while on duty or carrying out activities involving plants (Lee, 2019). Chang and Chen (2005) stated that workers showed high level of tension and vigilance when there were no natural landscapes or plants visible through the window in an office environment, and laid stress on natural elements and the need to create indoor garden spaces. Therefore, this study is to determine the components of agro-healing activities suitable for firefighters by surveying and analyzing the level of plant gardening activities of firefighters, distribution of quality of life according to demographic characteristics, preference of flowers and vegetables, plant color preference, and emotions according to plant color. Ultimately, the goal is to create an indoor garden, which is a natural environment that can alleviate stress and tension and improve the quality of life, and provide useful baseline data that can be applied to agro-healing activities.

\section{Research Methods}

\section{Characteristics of survey respondents}

To perform plant type/color preference and psychological assessment for gardening activities of firefighters in a high-risk occupational group, we conducted a survey on 117 fire officials in the fire department and three 119 regional centers located in Sunchang from March 20 to 31, 2020. The survey items were comprised of the level of plant gardening activities, SD (semantic differential) emotion scale, Korean version of WHOQOL-BREF, demographic characteristics, and preference of plant type and 
color for gardening. The demographic characteristics are as shown in Table 1.

\section{Assessment tools}

\section{Level of plant gardening activities}

The level of plant gardening activities scale is comprised of total 12 items rated on a 7-point Likert scale (Rural Development Administration[RDA], 2017), and the scale is comprised of 3 sub-domains. There are 4 items each for plant growing experience, plant preference tendency, and plant-related event, with higher scores indicating more plant growing experience or higher level of awareness. Cronbach's $\alpha$ in previous research was .890 in the online survey and .947 in the offline survey. Cronbach's $\alpha$ that indicates internal consistency of the scale used in this study was .832 for plant growing experience, .809 for plant preference tendency, and .923 for plant-related event.

\section{Korean version of WHOQOL-BREF}

We used the Korean version of WHOQOL-BREF to rate the quality of life of firefighters. The. WHOQOL-BREF is comprised of total 26 items: overall quality of life (2 items), physical health ( 7 items), psychological (6 items),

Table 1. Characteristic of survey respondents

\begin{tabular}{|c|c|c|c|}
\hline Variable & Categories & Frequency & Percent $(\%)$ \\
\hline \multirow{2}{*}{ Gender } & Male & 107 & 91.5 \\
\hline & Female & 10 & 8.5 \\
\hline \multirow{4}{*}{ Age } & $20-29 s$ & 17 & 14.7 \\
\hline & $30-39 s$ & 49 & 42.2 \\
\hline & $40-49 \mathrm{~s}$ & 26 & 22.4 \\
\hline & $50-59 \mathrm{~s}$ & 24 & 20.7 \\
\hline \multirow{3}{*}{ Education } & High school graduate & 24 & 20.5 \\
\hline & Univ. graduate & 88 & 75.2 \\
\hline & Postgraduate & 5 & 4.3 \\
\hline \multirow{4}{*}{$\begin{array}{c}\text { Monthly income } \\
\text { (Won) }\end{array}$} & $\leq 3,000,000$ & 51 & 43.6 \\
\hline & $3,010,000 \sim 4,000,000$ & 28 & 23.9 \\
\hline & $4,010,000 \sim 5,000,000$ & 16 & 13.7 \\
\hline & $\geq 5,010,000$ & 22 & 18.8 \\
\hline \multirow{3}{*}{ Marital status } & Married & 79 & 67.5 \\
\hline & Single & 36 & 30.8 \\
\hline & Divorce/bereavement/separation & 2 & 1.7 \\
\hline \multirow{4}{*}{ Work position } & Fire-fighter & 33 & 28.2 \\
\hline & Senior fire sergeant & 22 & 18.8 \\
\hline & Fire sergeant & 28 & 23.9 \\
\hline & $\geq$ Fire lieutenant & 34 & 29.1 \\
\hline \multirow{5}{*}{ Work type } & Fire suppression & 55 & 47.0 \\
\hline & Rescue & 13 & 11.1 \\
\hline & First aid & 24 & 20.5 \\
\hline & Administrations & 23 & 19.7 \\
\hline & 119 comprehensive disaster prevention center & 2 & 1.7 \\
\hline \multirow{3}{*}{ Shift pattern } & Regular daytime work & 25 & 21.4 \\
\hline & 3 rotation & 89 & 76.1 \\
\hline & The others & 3 & 2.5 \\
\hline
\end{tabular}

Note. $\mathrm{N}=117$. 
social relationships (3 items), and environment ( 8 items) (Min et al., 2000; Whoqol Group, 1998). Each item is rated on a 5 -point Likert scale $(1=$ Not at all, $2=$ Slightly, $3=$ Moderately, $4=$ Very, $5=$ Extremely), with higher total score of each domain indicating higher quality of life. Cronbach's $\alpha$ in previous research was .898, and that of each domain was .593 for overall quality of life, .777 for physical health, .713 for psychological, .583 for social relationships, and .765 for environment. Cronbach's $\alpha$ that indicates internal consistency of WHOQOL-BREF in this study was .972 . By domain, it was .818 for overall quality of life, .846 for physical health, .915 for psychological, .943 for social relationships, and .942 for environment.

\section{Type and color preference of flowering and vegetable plants}

We used images to survey plant type and color preferences. Types of flowering and vegetable plants selected were those that are accessible and easy to sow, transplant seedlings and manage for the general public in gardening activities. For plant color preference, we used images of foliage plants for green, and images of flowering plants for white, red, yellow, orange, and blue to be planted with foliage plants, thereby presenting total 6 colors to survey plant color preference. The preference survey items were rated on a 5-point Likert scale. Cronbach's $\alpha$ of the flowering plant preference in this study was .923, Cronbach's $\alpha$ of the vegetable plant preference was .884 , and Cronbach's $\alpha$ of plant color preference was .840 .

\section{Color psychology of plants using the semantic differential technique}

The semantic differential (SD) technique is widely used in assessing landscape, which is an element that not only changes in value depending on individual's subjective taste and preference, but is also difficult to quantify. This technique was developed by Osgood (1952) as a method to rate human emotions using adjectives, and is widely used in emotional or landscape assessment. This study used emotions toward plant colors in the survey with 13 pairs of emotion adjectives that can be used on plants, as well as adjectives for landscape assessment (Im, 2009). 13 ad- jectives were rated on a 5-point Likert scale, and the respondents were to mark the degree closest to their emotions (Jang et al., 2011, 2014). Cronbach's $\alpha$ of 13 pairs of emotion words in this study was .978 .

\section{Statistical analysis}

Data collected in this study were analyzed using IBM SPSS statistics 25. Level of plant gardening activities according to the demographic characteristics of firefighters, distribution of the Korean version of WHOQOL-BREF, the semantic differential technique of 13 pairs of words about plant color, and plant type and color preference were analyzed using one way ANOVA. The correlation between 13 pairs of emotion adjectives and color preference was analyzed using Pearson's correlation analysis, and demographic characteristics using frequency analysis.

\section{Results and Discussions}

\section{Analysis on psychological characteristics of firefighters in a high-risk occupational group}

\section{Plant experience and awareness according to the level of plant gardening activities}

As a result of surveying plant experience and awareness according to the level of plant gardening activities of firefighters, among the three factors (plant growing experience, plant preference tendency, and plant-related event), plantrelated event $(3.35 \pm 1.27)$ was highest, followed by plant preference tendency $(3.32 \pm 1.11)$ and plant growing experience $(3.16 \pm 1.25)$. We conducted Tukey's test for post-hoc analysis to test the statistical significance of plant experience and awareness by age and job position (Table 2, 3), and the difference between respondents in their $30 \mathrm{~s}$ and in their 50s about plant growing experience and plant preference tendency showed a statistically significant result $(p<.01)$. All three factors tended to show higher means among older respondents with higher job position. This suggests that the middle-aged group in their 50s have more plant growing experience or preference, as well as more memories and events about plants, compared to younger 
Table 2. Difference of three factors depending on ages using level of plant gardening activity by age of fire-fighter

\begin{tabular}{lcccccc}
\hline \multicolumn{1}{c}{ Variance } & $20 \mathrm{~s}$ & $30 \mathrm{~s}$ & $40 \mathrm{~s}$ & $50 \mathrm{~s}$ & $\mathrm{~F}$ & $p$ \\
\hline Plant growing experience & $3.00 \pm 1.18 \mathrm{ab}$ & $2.78 \pm 1.08 \mathrm{a}$ & $3.45 \pm 1.16 \mathrm{ab}$ & $3.78 \pm 1.44 \mathrm{~b}$ & 4.426 & $.006^{* *}$ \\
Plant preference tendency & $3.35 \pm 1.36 \mathrm{ab}$ & $3.04 \pm 1.04 \mathrm{a}$ & $3.38 \pm 0.91 \mathrm{ab}$ & $3.83 \pm 1.14 \mathrm{~b}$ & 2.892 & $.039^{*}$ \\
Plant-related event & $3.06 \pm 1.21 \mathrm{a}$ & $3.13 \pm 1.27 \mathrm{a}$ & $3.63 \pm 1.17 \mathrm{a}$ & $3.74 \pm 1.35 \mathrm{a}$ & 1.981 & .121 \\
\hline
\end{tabular}

Note. Values are mean \pm standard deviation $(\mathrm{N}=117)$. Mean separation within rows by Tukey's multiple range test, $5 \%$ level. $\stackrel{*}{p}<.05, \stackrel{* *}{p}<<.01$.

Table 3. Difference of three factors depending on ages using level of plant gardening activity by work position of firefighters

\begin{tabular}{llccccc}
\hline \multicolumn{1}{c}{ Variance } & Fire-fighter & Senior fire sergeant & Fire sergeant & ZFire lieutenant & $\mathrm{F}$ & $p$ \\
\hline Plant growing experience & $2.86 \pm 1.12 \mathrm{a}$ & $2.80 \pm 1.17 \mathrm{a}$ & $3.07 \pm 1.02 \mathrm{ab}$ & $3.76 \pm 1.40 \mathrm{~b}$ & 4.225 & $.007^{* *}$ \\
Plant preference tendency & $3.44 \pm 1.12 \mathrm{ab}$ & $2.92 \pm 1.14 \mathrm{a}$ & $2.90 \pm 0.87 \mathrm{a}$ & $3.81 \pm 1.07 \mathrm{~b}$ & 5.094 & $.002^{* *}$ \\
Plant-related event & $3.23 \pm 1.20 \mathrm{ab}$ & $2.97 \pm 1.26 \mathrm{a}$ & $3.22 \pm 1.17 \mathrm{ab}$ & $3.83 \pm 1.34 \mathrm{~b}$ & 2.609 & .055 \\
\hline
\end{tabular}

Note. Values are mean \pm standard deviation $(\mathrm{N}=117)$. Mean separation within rows by Tukey's multiple range test, $5 \%$ level. ${ }^{* *} p<.01$.

Table 4. Difference of three factors depending on ages using level of plant gardening activity by income of firefighters

\begin{tabular}{llccccc}
\hline \multicolumn{1}{c}{ Variance } & $\leq 3,000,000$ & $3,010,000 \sim 4,000,000$ & $4,010,000 \sim 5,000,000$ & $\geq 5,010,000$ & $\mathrm{~F}$ & $p$ \\
\hline Plant growing experience & $2.91 \pm 1.09 \mathrm{a}$ & $3.03 \pm 1.15 \mathrm{ab}$ & $3.14 \pm 1.20 \mathrm{ab}$ & $3.93 \pm 1.48 \mathrm{~b}$ & 3.893 & $.011^{*}$ \\
Plant preference tendency & $3.20 \pm 1.12 \mathrm{ab}$ & $3.25 \pm 1.05 \mathrm{ab}$ & $2.92 \pm 0.99 \mathrm{a}$ & $3.98 \pm 1.04 \mathrm{~b}$ & 3.743 & $.013^{*}$ \\
Plant-related event & $3.23 \pm 1.17 \mathrm{a}$ & $3.21 \pm 1.41 \mathrm{a}$ & $3.19 \pm 1.12 \mathrm{a}$ & $3.96 \pm 1.31 \mathrm{a}$ & 2.110 & .103 \\
\hline
\end{tabular}

Note. Values are mean \pm standard deviation $(\mathrm{N}=117)$. Mean separation within rows by Tukey's multiple range test, $5 \%$ level. ${ }^{*} p<.05$.

Table 5. Difference of three factors depending on ages using level of plant gardening activity by marital status of firefighters

\begin{tabular}{lccccc}
\hline \multicolumn{1}{c}{ Variance } & Married & Single & Divorce/bereavement/separation & F & $p$ \\
\hline Plant growing experience & $3.37 \pm 1.26 \mathrm{~b}$ & $2.78 \pm 1.12 \mathrm{a}$ & $1.88 \pm 0.53 \mathrm{a}$ & 4.049 & $.020^{*}$ \\
Plant preference tendency & $3.34 \pm 1.07 \mathrm{a}$ & $3.29 \pm 1.23 \mathrm{a}$ & $3.00 \pm 0.71 \mathrm{a}$ & .8 .108 & .897 \\
Plant-related event & $3.43 \pm 1.30 \mathrm{a}$ & $3.21 \pm 1.23 \mathrm{a}$ & $2.88 \pm 1.24 \mathrm{a}$ & .521 & .595 \\
\hline
\end{tabular}

Note. Values are mean \pm standard deviation $(\mathrm{N}=117)$. Mean separation within rows by Tukey's multiple range test, $5 \%$ level. ${ }^{*} p<.05$.

generations. In particular, there was a bigger difference with senior firefighters than fire lieutenants with high job position. This result is similar to Jang et al. (2019) who reported that there was more plant growing experience or plant preference, as well as more memories or events related to plants, among 50s and above than 20-40s that are younger.

As a result of examining plant experience and awareness by average monthly income, all factors showed highest scores in the group that had a monthly income of KRW
5.01 million or higher. There was a statistically significant result between the group that had a monthly income of KRW 5.01 million or higher and the group that had a monthly income of KRW 3 million or lower for plant growing experience, and in the group that had a monthly income of KRW 3.01-4 million for plant preference tendency (Table 4). Moreover, by marital status of firefighters, plant growing experience showed higher scores in the married group than the singles group, showing statistical significance (Table 5). We also examined the difference in plant experi- 
Table 6. Difference of three factors depending on ages using level of plant gardening activity by work type of firefighters

\begin{tabular}{llcccccc}
\hline \multicolumn{1}{c}{ Variance $^{\mathrm{z}}$} & $\mathrm{A}$ & $\mathrm{B}$ & $\mathrm{C}$ & $\mathrm{D}$ & $\mathrm{E}$ & $\mathrm{F}$ & $p$ \\
\hline Plant growing experience & $3.03 \pm 1.18 \mathrm{ab}$ & $3.85 \pm 0.76 \mathrm{~b}$ & $2.59 \pm 1.23 \mathrm{a}$ & $3.62 \pm 1.38 \mathrm{~b}$ & $3.88 \pm 0.53 \mathrm{a}$ & 3.627 & $.008^{* *}$ \\
Plant preference tendency & $3.29 \pm 1.09 \mathrm{a}$ & $3.71 \pm 0.69 \mathrm{a}$ & $2.86 \pm 1.17 \mathrm{a}$ & $3.64 \pm 1.18 \mathrm{a}$ & $3.50 \pm 1.06 \mathrm{a}$ & 1.990 & .101 \\
Plant-related event & $3.23 \pm 1.22 \mathrm{a}$ & $3.68 \pm 1.10 \mathrm{a}$ & $2.94 \pm 1.38 \mathrm{a}$ & $3.87 \pm 1.26 \mathrm{a}$ & $3.75 \pm 1.06 \mathrm{a}$ & 2.062 & .091 \\
\hline
\end{tabular}

Note. Values are mean \pm standard deviation $(\mathrm{N}=117)$. Mean separation within rows by Tukey's multiple range test, $5 \%$ level. $\mathrm{A}=$ Fire suppression; $\mathrm{B}=$ Rescue; $\mathrm{C}=$ First aid $\mathrm{D}=$ Administrations; $\mathrm{E}=119$ comprehensive disaster prevention center. ${ }^{* *} p<.01$.

Table 7. Differences in world health organization quality of life assessment instrument (WHOQOL-BREF) by age of firefighters

\begin{tabular}{lcccccc}
\hline \multicolumn{1}{c}{ Variance } & $20 \mathrm{~s}$ & $30 \mathrm{~s}$ & $40 \mathrm{~s}$ & $50 \mathrm{~s}$ & $\mathrm{~F}$ & $p$ \\
\hline Overall quality of life & $3.71 \pm 1.02 \mathrm{~b}$ & $3.48 \pm 0.80 \mathrm{ab}$ & $3.37 \pm 0.71 \mathrm{ab}$ & $2.96 \pm 0.79 \mathrm{a}$ & 3.301 & $.023^{*}$ \\
Physical health & $3.98 \pm 0.71 \mathrm{~b}$ & $3.77 \pm 0.64 \mathrm{~b}$ & $3.64 \pm 0.66 \mathrm{ab}$ & $3.26 \pm 0.65 \mathrm{a}$ & 4.760 & $.004^{* *}$ \\
Psychological health & $3.88 \pm 0.89 \mathrm{~b}$ & $3.70 \pm 0.62 \mathrm{ab}$ & $3.59 \pm 0.69 \mathrm{ab}$ & $3.17 \pm 0.83 \mathrm{a}$ & 3.952 & $.010^{*}$ \\
Social relationships & $3.88 \pm 0.93 \mathrm{~b}$ & $3.58 \pm 0.83 \mathrm{~b}$ & $3.45 \pm 0.76 \mathrm{ab}$ & $2.91 \pm 0.85 \mathrm{a}$ & 5.260 & $.002^{* *}$ \\
Environment & $3.82 \pm 0.97 \mathrm{~b}$ & $3.45 \pm 0.74 \mathrm{ab}$ & $3.28 \pm 0.70 \mathrm{ab}$ & $2.93 \pm 0.75 \mathrm{a}$ & 4.904 & $.003^{* *}$ \\
Total & $3.88 \pm 0.83 \mathrm{~b}$ & $3.61 \pm 0.65 \mathrm{~b}$ & $3.47 \pm 0.64 \mathrm{ab}$ & $3.08 \pm 0.69 \mathrm{a}$ & 5.227 & $.002^{* *}$ \\
\hline
\end{tabular}

Note. Values are mean \pm standard deviation $(\mathrm{N}=117)$. Mean separation within rows by Tukey's multiple range test, $5 \%$ level. $\stackrel{*}{p}<.05, \stackrel{* *}{p}<<.01$.

ence and awareness depending on the current duty, and found that plant growing experience showed higher scores in the rescue and administration groups than the first aid group, with statistical significance (Table 6). The statistical difference could not be found in the 119 comprehensive disaster prevention center group due to the small population size. Therefore, the difference in the level of plant gardening activities tended to be greater by age, with higher job position and income, and among the married groups. There has been hardly any research on the level of plant growing experiences targeting firefighters, and it seems that it is necessary to survey plant experience or awareness of firefighters based on continuous research.

\section{Korean version of WHOQOL-BREF}

As a result of examining the difference in quality of life according to demographic characteristics of firefighters in a high-risk occupational group through WHOQOLBREF, it was found that the average quality of life of 117 participants was $3.51 \pm 0.72$. Moreover, we analyzed the five sub-domains such as overall quality of life, physical health, psychological, social relationships, and environment according to demographic characteristics. The results showed that there was statistical significance in all of the five sub-domains among groups by age and job position, and not by academic background (Table 7-9). The scores of overall quality of life, psychological, and environment were higher among the 20s than 50s, and the scores of physical health and social relationships were higher among the 20s-30s than 50s (Table 8). By job position, the scores of all 5 sub-domains were higher among the firefighter position than fire lieutenant or higher (Table 9). By average monthly income, the scores of only physical health were higher in the income group of KRW 3 million or lower than the income group of KRW 5.01 million or higher, showing statistical significance (Table 10). By marital status (Table 11), the scores were highest overall in the divorced/bereaved/separated group but had no statistical significance, and the scores of physical health, social relationships, and environment were higher in the singles group than the married group, showing statistical significance $(p<.05)$. By current duty, the scores of 4 sub-domains except psychological were lower in administration than rescue, first aid, and the 119 comprehensive disaster prevention center but had no statistical significance. The scores of overall quality of life, physical health, social relationships, 
Table 8. Differences in world health organization quality of life assessment instrument (WHOQOL-BREF) by work position of firefighters

\begin{tabular}{lcccccc}
\hline \multicolumn{1}{c}{ Variance } & Fire-fighter & Senior fire sergeant & Fire sergeant & \Fire lieutenant & F & $p$ \\
\hline Overall quality of life & $3.76 \pm 0.90 \mathrm{~b}$ & $3.36 \pm 0.64 \mathrm{ab}$ & $3.34 \pm 0.72 \mathrm{ab}$ & $3.07 \pm 0.86 \mathrm{a}$ & 4.087 & $.009^{* *}$ \\
Physical health & $4.03 \pm 0.64 \mathrm{~b}$ & $3.52 \pm 0.56 \mathrm{~b}$ & $3.62 \pm 0.66 \mathrm{ab}$ & $3.45 \pm 0.71 \mathrm{a}$ & 5.095 & $.002^{* *}$ \\
Psychological health & $3.92 \pm 0.70 \mathrm{~b}$ & $3.48 \pm 0.54 \mathrm{ab}$ & $3.63 \pm 0.73 \mathrm{ab}$ & $3.35 \pm 0.84 \mathrm{a}$ & 3.712 & $.014^{*}$ \\
Social relationships & $3.90 \pm 0.87 \mathrm{~b}$ & $3.33 \pm 0.63 \mathrm{ab}$ & $3.46 \pm 0.84 \mathrm{ab}$ & $3.11 \pm 0.91 \mathrm{a}$ & 5.192 & $.002^{* *}$ \\
Environment & $3.77 \pm 0.85 \mathrm{~b}$ & $3.34 \pm 0.59 \mathrm{ab}$ & $3.25 \pm 0.75 \mathrm{ab}$ & $3.09 \pm 0.81 \mathrm{a}$ & 4.613 & $.004^{* *}$ \\
Total & $3.89 \pm 0.72 \mathrm{~b}$ & $3.42 \pm 0.52 \mathrm{ab}$ & $3.47 \pm 0.68 \mathrm{ab}$ & $3.25 \pm 0.75 \mathrm{a}$ & 5.142 & $.002^{* *}$ \\
\hline
\end{tabular}

Note. Values are mean \pm standard deviation $(\mathrm{N}=117)$. Mean separation within rows by Tukey's multiple range test, $5 \%$ level.

${ }^{*} p<.05,{ }^{* *} p<.01$.

Table 9. Differences in pworld health organization quality of life assessment instrument (WHOQOL-BREF) by education background of firefighters

\begin{tabular}{lccccc}
\hline \multicolumn{1}{c}{ Variance } & High school graduate & Univ. graduate & Postgraduate & F & $p$ \\
\hline Overall quality of life & $3.02 \pm 0.90 \mathrm{a}$ & $3.47 \pm 0.79 \mathrm{a}$ & $3.60 \pm 0.96 \mathrm{a}$ & 3.011 & $.053^{\mathrm{NS}}$ \\
Physical health & $3.58 \pm 0.77 \mathrm{a}$ & $3.69 \pm 0.66 \mathrm{a}$ & $3.74 \pm 0.79 \mathrm{a}$ & 0.251 & $.779^{\text {NS }}$ \\
Psychological health & $3.41 \pm 0.91 \mathrm{a}$ & $3.64 \pm 0.71 \mathrm{a}$ & $3.77 \pm 0.57 \mathrm{a}$ & 1.030 & $.360^{\mathrm{NS}}$ \\
Social relationships & $3.20 \pm 0.92 \mathrm{a}$ & $3.53 \pm 0.87 \mathrm{a}$ & $3.53 \pm 0.77 \mathrm{a}$ & 1.321 & $.271^{\mathrm{NS}}$ \\
Environment & $3.21 \pm 0.93 \mathrm{a}$ & $3.40 \pm 0.79 \mathrm{a}$ & $3.45 \pm 0.52 \mathrm{a}$ & 0.576 & $.564^{\mathrm{NS}}$ \\
Total & $3.34 \pm 0.82 \mathrm{a}$ & $3.55 \pm 0.70 \mathrm{a}$ & $3.62 \pm 0.58 \mathrm{a}$ & 0.878 & $.418^{\mathrm{NS}}$ \\
\hline
\end{tabular}

Note. Values are mean \pm standard deviation $(\mathrm{N}=117)$. Mean separation within rows by Tukey's multiple range test, $5 \%$ level.

${ }^{\mathrm{NS}}$ Non-significant

Table 10. Differences in world health organization quality of life assessment instrument (WHOQOL-BREF) by income of firefighters

\begin{tabular}{lcccccc}
\hline \multicolumn{1}{c}{ Variance } & $\leq 3,000,000$ & $3,010,000 \sim 4,000,000$ & $4,010,000 \sim 5,000,000$ & $\geq 5,010,000$ & $\mathrm{~F}$ & $p$ \\
\hline Overall quality of life & $3.54 \pm 0.90 \mathrm{a}$ & $3.43 \pm 0.70 \mathrm{a}$ & $3.19 \pm 0.96 \mathrm{a}$ & $3.11 \pm 0.69 \mathrm{a}$ & 1.702 & .171 \\
Physical health & $3.87 \pm 0.63 \mathrm{a}$ & $3.60 \pm 0.74 \mathrm{a}$ & $3.46 \pm 0.80 \mathrm{a}$ & $3.44 \pm 0.55 \mathrm{a}$ & 3.078 & $.030^{*}$ \\
Psychological health & $3.70 \pm 0.68 \mathrm{a}$ & $3.72 \pm 0.78 \mathrm{a}$ & $3.39 \pm 0.89 \mathrm{a}$ & $3.38 \pm 0.73 \mathrm{a}$ & 1.597 & .194 \\
Social relationships & $3.59 \pm 0.84 \mathrm{a}$ & $3.58 \pm 0.91 \mathrm{a}$ & $3.26 \pm 0.99 \mathrm{a}$ & $3.15 \pm 0.77 \mathrm{a}$ & 1.765 & .158 \\
Environment & $3.48 \pm 0.84 \mathrm{a}$ & $3.44 \pm 0.84 \mathrm{a}$ & $3.27 \pm 0.79 \mathrm{a}$ & $3.09 \pm 0.65 \mathrm{a}$ & 1.381 & .252 \\
Total & $3.65 \pm 0.71 \mathrm{a}$ & $3.56 \pm 0.74 \mathrm{a}$ & $3.34 \pm 0.80 \mathrm{a}$ & $3.26 \pm 0.59 \mathrm{a}$ & 1.946 & .126 \\
\hline
\end{tabular}

Note. Values are mean \pm standard deviation $(\mathrm{N}=117)$. Mean separation within rows by Tukey's multiple range test, $5 \%$ level. ${ }^{*} p<.05$.

Table 11. Differences in world health organization quality of life assessment instrument (WHOQOL-BREF) by marital status of firefighters

\begin{tabular}{lccccc}
\hline \multicolumn{1}{c}{ Variance } & Married & Single & Divorce/bereavement/separation & $\mathrm{F}$ & $p$ \\
\hline Overall quality of life & $3.26 \pm 0.74 \mathrm{a}$ & $3.64 \pm 0.99 \mathrm{a}$ & $3.75 \pm 0.35 \mathrm{a}$ & 2.823 & .064 \\
Physical health & $3.53 \pm 0.65 \mathrm{a}$ & $3.96 \pm 0.70 \mathrm{~b}$ & $3.93 \pm 0.51 \mathrm{ab}$ & 5.292 & $.006^{* *}$ \\
Psychological health & $3.49 \pm 0.73 \mathrm{a}$ & $3.80 \pm 0.78 \mathrm{a}$ & $4.18 \pm 0.02 \mathrm{a}$ & 2.827 & .063 \\
Social relationships & $3.30 \pm 0.81 \mathrm{a}$ & $3.77 \pm 0.94 \mathrm{~b}$ & $4.17 \pm 0.24 \mathrm{ab}$ & 4.407 & $.014^{*}$ \\
Environment & $3.18 \pm 0.70 \mathrm{a}$ & $3.72 \pm 0.90 \mathrm{~b}$ & $4.13 \pm 0.71 \mathrm{ab}$ & 7.094 & $<.001^{* * *}$ \\
Total & $3.37 \pm 0.65 \mathrm{a}$ & $3.80 \pm 0.79 \mathrm{~b}$ & $4.06 \pm 0.03 \mathrm{ab}$ & 5.481 & $.005^{* *}$ \\
\hline
\end{tabular}

Note. Values are mean \pm standard deviation $(\mathrm{N}=117)$. Mean separation within rows by Tukey's multiple range test, $5 \%$ level.

${ }^{*} p<.05, \stackrel{* *}{p}<.01, \stackrel{* * *}{p}<<.001$. 
Table 12. Differences in world health organization quality of life assessment instrument (WHOQOL-BREF) by work type of firefighters

\begin{tabular}{lccccccc}
\hline \multicolumn{1}{c}{ Variance } & $\mathrm{A}^{\mathrm{z}}$ & $\mathrm{B}$ & $\mathrm{C}$ & $\mathrm{D}$ & $\mathrm{E}$ & $\mathrm{F}$ & $p$ \\
\hline Overall quality of life & $3.54 \pm 0.88 \mathrm{~b}$ & $3.38 \pm 0.62 \mathrm{ab}$ & $3.50 \pm 0.79 \mathrm{ab}$ & $2.96 \pm 0.75 \mathrm{a}$ & $2.75 \pm 1.06 \mathrm{ab}$ & 2.480 & $.048^{*}$ \\
Physical health & $3.81 \pm 0.68 \mathrm{~b}$ & $3.62 \pm 0.52 \mathrm{ab}$ & $3.77 \pm 0.68 \mathrm{ab}$ & $3.31 \pm 0.70 \mathrm{a}$ & $3.21 \pm 0.71 \mathrm{ab}$ & 2.623 & $.039^{*}$ \\
Psychological health & $3.72 \pm 0.74 \mathrm{a}$ & $3.51 \pm 0.62 \mathrm{a}$ & $3.71 \pm 0.65 \mathrm{a}$ & $3.29 \pm 0.88 \mathrm{a}$ & $3.08 \pm 0.59 \mathrm{a}$ & 1.775 & .139 \\
Social relationships & $3.62 \pm 0.90 \mathrm{a}$ & $3.31 \pm 0.55 \mathrm{a}$ & $3.67 \pm 0.80 \mathrm{a}$ & $3.04 \pm 0.88 \mathrm{a}$ & $2.50 \pm 1.18 \mathrm{a}$ & 2.941 & $.024^{*}$ \\
Environment & $3.55 \pm 0.89 \mathrm{~b}$ & $3.05 \pm 0.67 \mathrm{ab}$ & $3.55 \pm 0.67 \mathrm{ab}$ & $2.95 \pm 0.63 \mathrm{a}$ & $3.13 \pm 0.53 \mathrm{ab}$ & 3.345 & $.013^{*}$ \\
Total & $3.66 \pm 0.75 \mathrm{~b}$ & $3.36 \pm 0.55 \mathrm{ab}$ & $3.65 \pm 0.65 \mathrm{ab}$ & $3.14 \pm 0.69 \mathrm{a}$ & $3.04 \pm 0.71 \mathrm{ab}$ & 2.953 & $.023^{*}$ \\
\hline
\end{tabular}

Note. Values are mean \pm standard deviation $(\mathrm{N}=117)$. Mean separation within rows by Tukey's multiple range test, $5 \%$ level. $\mathrm{A}=$ Fire suppression; $\mathrm{B}=$ Rescue $\mathrm{C}=$ First aid $\mathrm{D}=$ Administrations; $\mathrm{E}=119$ comprehensive disaster prevention center. ${ }^{*} p<.05$.

Table 13. The difference of emotional words between the six colors of plant

\begin{tabular}{|c|c|c|c|c|c|c|c|c|}
\hline \multirow{2}{*}{$\mathrm{V}^{\mathrm{z}}$} & \multicolumn{6}{|c|}{ Treatments (color) } & \multirow{2}{*}{$\mathrm{F}$} & \multirow{2}{*}{$p$} \\
\hline & White & Green & Red & Yellow & Orange & Blue & & \\
\hline $\mathrm{B} 1$ & $4.03 \pm 1.16 \mathrm{~cd}$ & $2.66 \pm 1.09 \mathrm{a}$ & $3.59 \pm 1.25 \mathrm{bc}$ & $4.25 \pm 0.98 \mathrm{~d}$ & $3.78 \pm 1.17 \mathrm{bc}$ & $3.33 \pm 1.30 \mathrm{~b}$ & 24.330 & $<.001^{* * *}$ \\
\hline $\mathrm{B} 2$ & $3.99 \pm 1.10 \mathrm{c}$ & $2.79 \pm 1.05 \mathrm{a}$ & $3.25 \pm 1.20 \mathrm{~b}$ & $4.01 \pm 1.08 \mathrm{c}$ & $3.63 \pm 1.14 b c$ & $3.37 \pm 1.24 b$ & 17.777 & $<.001^{* * *}$ \\
\hline B3 & $3.78 \pm 1.18 \mathrm{c}$ & $2.86 \pm 0.96 \mathrm{a}$ & $2.95 \pm 1.12 \mathrm{a}$ & $3.82 \pm 1.04 \mathrm{c}$ & $3.47 \pm 1.16 b c$ & $3.24 \pm 1.24 \mathrm{ab}$ & 13.743 & $<.001^{* * *}$ \\
\hline B4 & $3.42 \pm 1.17 \mathrm{~b}$ & $2.80 \pm 1.06 \mathrm{a}$ & $3.43 \pm 1.11 \mathrm{~b}$ & $3.98 \pm 1.02 \mathrm{c}$ & $3.74 \pm 1.08 b c$ & $2.88 \pm 1.29 \mathrm{a}$ & 17.530 & $<.001^{* * *}$ \\
\hline B5 & $3.56 \pm 1.09 \mathrm{~b}$ & $3.13 \pm 1.11 \mathrm{ab}$ & $2.90 \pm 1.05 \mathrm{a}$ & $3.53 \pm 1.01 \mathrm{~b}$ & $3.26 \pm 1.01 \mathrm{ab}$ & $3.52 \pm 1.20 \mathrm{~b}$ & 6.269 & $<.001^{* * *}$ \\
\hline B6 & $3.27 \pm 1.03 \mathrm{bc}$ & $2.87 \pm 0.79 \mathrm{ab}$ & $2.67 \pm 1.27 \mathrm{a}$ & $3.37 \pm 1.03 \mathrm{c}$ & $3.16 \pm 1.05 b c$ & $3.15 \pm 1.16 b c$ & 6.256 & $<.001^{* * *}$ \\
\hline B7 & $3.52 \pm 1.15 \mathrm{a}$ & $3.28 \pm 1.05 \mathrm{a}$ & $3.27 \pm 1.15 \mathrm{a}$ & $3.63 \pm 1.04 \mathrm{a}$ & $3.43 \pm 1.05 \mathrm{a}$ & $3.33 \pm 1.13 \mathrm{a}$ & 1.745 & .122 \\
\hline B8 & $3.59 \pm 1.12 b c$ & $2.98 \pm 0.91 \mathrm{a}$ & $3.47 \pm 1.03 \mathrm{bc}$ & $3.87 \pm 1.05 \mathrm{c}$ & $3.60 \pm 1.00 \mathrm{bc}$ & $3.24 \pm 1.16 \mathrm{ab}$ & 9.000 & $<.001^{* * *}$ \\
\hline B9 & $3.79 \pm 1.10 \mathrm{~b}$ & $3.29 \pm 0.93 \mathrm{a}$ & $3.13 \pm 1.09 \mathrm{a}$ & $3.75 \pm 1.02 \mathrm{~b}$ & $3.46 \pm 1.01 \mathrm{ab}$ & $3.40 \pm 1.09 \mathrm{ab}$ & 6.335 & $<.001^{* * *}$ \\
\hline B10 & $3.88 \pm 1.15 \mathrm{c}$ & $3.18 \pm 0.93 \mathrm{a}$ & $3.40 \pm 1.11 \mathrm{ab}$ & $3.82 \pm 1.03 b c$ & $3.57 \pm 1.04 \mathrm{abc}$ & $3.45 \pm 1.17 \mathrm{abc}$ & 6.189 & $<.001^{* * *}$ \\
\hline B11 & $3.80 \pm 1.06 \mathrm{c}$ & $3.42 \pm 1.00 \mathrm{bc}$ & $2.94 \pm 1.20 \mathrm{a}$ & $3.57 \pm 1.01 b c$ & $3.36 \pm 1.05 \mathrm{ab}$ & $3.51 \pm 1.12 b c$ & 7.245 & $<.001^{* * *}$ \\
\hline $\mathrm{B} 12$ & $3.89 \pm 1.07 \mathrm{c}$ & $3.39 \pm 0.96 \mathrm{ab}$ & $3.09 \pm 1.06 \mathrm{a}$ & $3.72 \pm 1.02 b c$ & $3.48 \pm 1.00 \mathrm{ab}$ & $3.56 \pm 1.12 b c$ & 7.477 & $<.001^{* * *}$ \\
\hline B13 & $3.65 \pm 1.14 \mathrm{bc}$ & $2.68 \pm 1.03 \mathrm{a}$ & $3.85 \pm 1.16 \mathrm{c}$ & $3.83 \pm 1.13 \mathrm{c}$ & $3.69 \pm 1.01 b c$ & $3.28 \pm 1.17 \mathrm{~b}$ & 17.025 & $<.001^{* * *}$ \\
\hline
\end{tabular}

Note. Values are mean \pm standard deviation $(\mathrm{N}=117)$. Mean separation within rows by Tukey's multiple range test, $5 \%$ level.

${ }^{\mathrm{z}} \mathrm{V}$ = Variance; B1 = dark-bright; B2 = dusky-clean; B3 = heavy-light; B4 = cool-warm; B5 = stuffy-fresh; B6 = strong-pale;

$\mathrm{B} 7=$ distracted-concentrated; B8 = depressed-spirited; B9 = nervous-relaxed; B10 = unplesant-plesant; B11 = vibrant-calm;

$\mathrm{B} 12$ = uneasy-comfortable; B13 = modes-fancy.

${ }^{* * *} p<.001$.

and environment were higher in fire suppression than administration, showing statistical significance (Table 12). The results of this study that the quality of life was higher in the singles group than married, 20s than 50s, firefighter position than fire lieutenant or higher, and on-site duties such as first aid and 19 comprehensive disaster prevention center than administration are supported by Yun and Hong (2018) and Lee (2019), who reported that the quality of life according to the general characteristics of 119 ambulance workers was higher in singles than married, age be- low 30 than above, firefighter position than higher job positions like senior firefighter or fire lieutenant, and first aid duty than administration.

\section{Emotional responses of firefighters to 6 plant colors}

As a result of analyzing the emotional responses of firefighters to 6 plant colors using 13 pairs of emotion adjectives that can be expressed in relation to plants (Table 13 and Fig. 1), it was found that the white group showed 
high scores in 'bright', 'clean', 'comfortable', the green group in 'calm', 'comfortable', 'relaxed', the red group in 'fancy', 'bright', 'spirited', the yellow group in 'bright', 'clean', 'warm', the orange group in 'bright', 'clean', 'fancy', and the blue group in 'comfortable', 'calm', 'cool'. This result of this study that the white, green and blue groups felt calm and comfortable, the red and orange groups felt fancy,

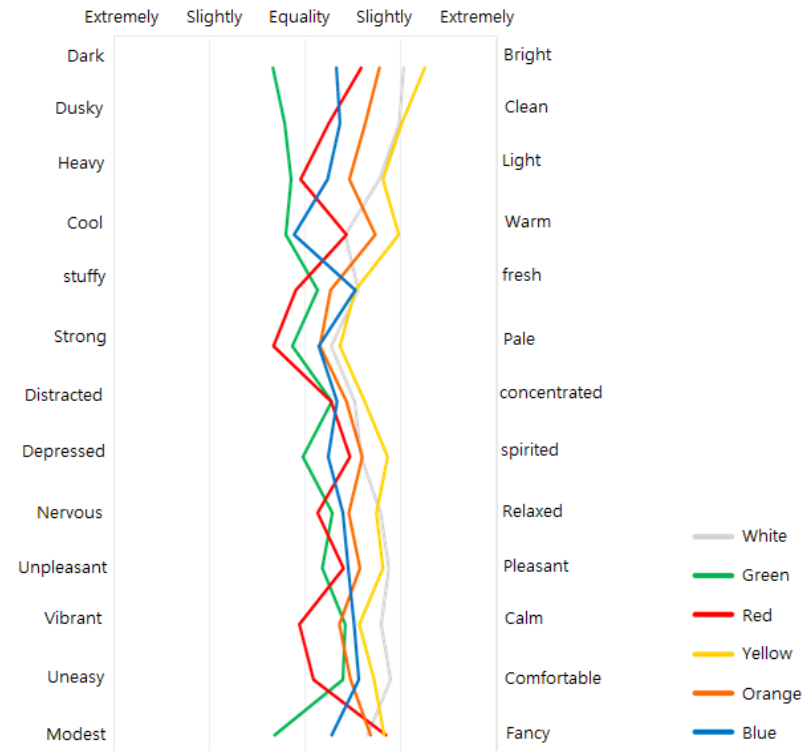

Fig. 1. The difference of emotional words between the six colors of plant

Table 14. The preference of garden plants.

\begin{tabular}{|c|c|c|c|c|c|c|c|c|c|}
\hline & \multirow{2}{*}{ Species } & \multirow{2}{*}{$\mathrm{N}$} & \multirow{2}{*}{ Mean $^{\mathrm{z}}$} & \multirow{2}{*}{$\mathrm{SD}$} & \multicolumn{3}{|c|}{ Tukey's test $\mathrm{a}=.05$} & \multirow{2}{*}{$\mathrm{F}$} & \multirow{2}{*}{$p$} \\
\hline & & & & & 1 & 2 & 3 & & \\
\hline \multirow{12}{*}{ Preference } & Pelargonium spp. & 117 & 2.97 & 0.93 & $\mathrm{a}^{\mathrm{y}}$ & & & \multirow{12}{*}{4.362} & \multirow{12}{*}{$<.001^{* * *}$} \\
\hline & Vinca rosea & 116 & 3.14 & 0.94 & $\mathrm{a}$ & $\mathrm{b}$ & & & \\
\hline & Salvia elegans & 117 & 3.20 & 0.88 & $\mathrm{a}$ & $\mathrm{b}$ & & & \\
\hline & Ocimum basilicum & 117 & 3.25 & 0.90 & $\mathrm{a}$ & $\mathrm{b}$ & & & \\
\hline & Stevia rebaudiana & 117 & 3.26 & 0.83 & $\mathrm{a}$ & $\mathrm{b}$ & & & \\
\hline & Gomphrena globosa & 117 & 3.29 & 0.97 & $\mathrm{a}$ & $\mathrm{b}$ & & & \\
\hline & Tropaeolum majus & 117 & 3.29 & 0.92 & $\mathrm{a}$ & $\mathrm{b}$ & & & \\
\hline & Zinnia elegans & 117 & 3.32 & 0.93 & $\mathrm{a}$ & $\mathrm{b}$ & $\mathrm{c}$ & & \\
\hline & Calendula officinalis & 117 & 3.39 & 0.89 & & $\mathrm{~b}$ & $\mathrm{c}$ & & \\
\hline & Mentha piperita & 117 & 3.39 & 0.87 & & $\mathrm{~b}$ & $\mathrm{c}$ & & \\
\hline & Lavandula spp. & 117 & 3.47 & 0.97 & & $\mathrm{~b}$ & $\mathrm{c}$ & & \\
\hline & Rosmarinus officinalis L. & 117 & 3.68 & 0.90 & & & $\mathrm{c}$ & & \\
\hline
\end{tabular}

${ }^{\mathrm{z}} 5$-point Likert scale was used, where $1=$ never preference; $5=$ very preference.

${ }^{\mathrm{y}}$ Mean separation within columns by Tukey's multiple range test, $5 \%$ level $(\mathrm{n}=117)$.

Journal of People, Plants, and Environment Vol. 23, No. 5, 2020.529 and the yellow group felt warm is supported by Jang et al. (2015) stating that the green and white groups generally showed high scores in 'comfortable', 'calm', and the red and yellow groups in 'warm', 'fancy'. It is also supported by Lee and Son (1999) that people felt comfortable and stable in visual stimulation of green plants, and by Song (2004) who claimed that people felt more comfortable in a space with green plants.

\section{Plant and color preference of firefighters}

The firefighters were to respond their preference in plant type and color for creation of an indoor healing garden and agro-healing activities on a 5-point Likert scale, and the Tukey's multiple range test was conducted for posttest. The results showed that most of them showed high preference in herbal plants among the 12 types of flowering plants, in the order of Rosmarinus officinalis $>$ Lavandula spp. > Mentha piperita, Calendula officinalis. Pelargonium spp. showed the least preference among the flowering plants (Table 14). Among the 12 vegetable plants, fruit vegetable Lycopersicon esculentum showed the highest preference, followed by Lactuca sativa $>$ Allium tuberosum $>$ Chrysanthemum coronarium L. > Brassica oleracea $>$ Brassica campestris $>$ Perilla frutescens $>$ Capsicum annuum L. > Solanum melongena $>$ Beta vulgaris $>$ Raphanus 
Table 15. The preference of vegetable plants

\begin{tabular}{|c|c|c|c|c|c|c|c|c|c|c|c|c|c|}
\hline & \multirow{2}{*}{ Species } & \multirow{2}{*}{$\mathrm{N}$} & \multirow{2}{*}{ Mean $^{2}$} & \multirow{2}{*}{$\mathrm{SD}$} & \multicolumn{7}{|c|}{ Tukey's test $\mathrm{a}=0.05$} & \multirow{2}{*}{$\mathrm{F}$} & \multirow{2}{*}{$p$} \\
\hline & & & & & 1 & 2 & 3 & 4 & 5 & 6 & 7 & & \\
\hline \multirow{12}{*}{ Preference } & Angelica acutiloba & 115 & 2.99 & 1.00 & $\mathrm{a}^{\mathrm{y}}$ & & & & & & & \multirow{12}{*}{12.980} & \multirow{12}{*}{$<.001^{* * *}$} \\
\hline & Raphanus sativus & 116 & 3.12 & 0.86 & $\mathrm{a}$ & $\mathrm{b}$ & & & & & & & \\
\hline & Beta vulgaris & 115 & 3.16 & 0.95 & $\mathrm{a}$ & $\mathrm{b}$ & $\mathrm{c}$ & & & & & & \\
\hline & Solanum melongena & 116 & 3.29 & 1.06 & $\mathrm{a}$ & $\mathrm{b}$ & $\mathrm{c}$ & d & & & & & \\
\hline & Capsicum anпиит $\mathrm{L}$. & 116 & 3.41 & 0.95 & $\mathrm{a}$ & $\mathrm{b}$ & $\mathrm{c}$ & d & $\mathrm{e}$ & & & & \\
\hline & Perilla frutescens & 116 & 3.50 & 1.05 & & $\mathrm{~b}$ & $\mathrm{c}$ & d & $\mathrm{e}$ & $\mathrm{f}$ & & & \\
\hline & Brassica campestris & 116 & 3.51 & 1.03 & & $\mathrm{~b}$ & $\mathrm{c}$ & d & $\mathrm{e}$ & $\mathrm{f}$ & & & \\
\hline & Brassica oleracea & 116 & 3.55 & 1.00 & & & $\mathrm{c}$ & d & $\mathrm{e}$ & $\mathrm{f}$ & & & \\
\hline & Chrysanthemum coronarium $\mathrm{L}$. & 115 & 3.66 & 0.97 & & & & $\mathrm{~d}$ & $\mathrm{e}$ & $\mathrm{f}$ & & & \\
\hline & Allium tuberosum & 116 & 3.73 & 0.92 & & & & & $\mathrm{e}$ & $\mathrm{f}$ & $\mathrm{g}$ & & \\
\hline & Lactuca sativa & 115 & 3.91 & 0.89 & & & & & & $\mathrm{f}$ & $\mathrm{g}$ & & \\
\hline & Lycopersicon esculentum & 115 & 4.09 & 0.96 & & & & & & & $\mathrm{~g}$ & & \\
\hline
\end{tabular}

${ }^{\mathrm{z}} 5$-point Likert scale was used, where $1=$ never preference; $5=$ very preference.

${ }^{\mathrm{y}}$ Mean separation within columns by Tukey's multiple range test, $5 \%$ level $(\mathrm{n}=117)$.

Table 16. The color preference of garden plants

\begin{tabular}{|c|c|c|c|c|c|c|c|c|c|}
\hline & \multirow{2}{*}{ Color } & \multirow{2}{*}{$\mathrm{N}$} & \multirow{2}{*}{ Mean $^{\mathrm{z}}$} & \multirow{2}{*}{$\mathrm{SD}$} & \multicolumn{3}{|c|}{ Tukey's test $\mathrm{a}=0.05$} & \multirow{2}{*}{$\mathrm{F}$} & \multirow{2}{*}{$p$} \\
\hline & & & & & 1 & 2 & 3 & & \\
\hline \multirow{6}{*}{ Preference } & Green & 116 & 2.97 & 0.74 & $a^{y}$ & & & \multirow{6}{*}{11.101} & \multirow{6}{*}{$<.001^{* * *}$} \\
\hline & Red & 116 & 3.39 & 0.91 & & $\mathrm{~b}$ & & & \\
\hline & Orange & 113 & 3.56 & 0.88 & & $\mathrm{~b}$ & $\mathrm{c}$ & & \\
\hline & Blue & 114 & 3.57 & 0.97 & & $\mathrm{~b}$ & $\mathrm{c}$ & & \\
\hline & White & 115 & 3.63 & 0.87 & & $\mathrm{~b}$ & $\mathrm{c}$ & & \\
\hline & Yellow & 116 & 3.74 & 0.88 & & & $\mathrm{c}$ & & \\
\hline
\end{tabular}

${ }^{\mathrm{z}} 5$-point Likert scale was used, where $1=$ never preference; $5=$ very preference.

${ }^{\mathrm{y}}$ Mean separation within columns by Tukey's multiple range test, $5 \%$ level $(\mathrm{n}=117)$.

sativus >Angelica acutiloba, with statistical significance (Table 15). As a result of examining the preference of 6 plant colors, F-value was 11.101 and the significance probability was .001 , showing that yellow was most preferred, followed by white, blue, orange, red, and green, with a statistically significant difference (Table 16). Green was least preferred in this study, contrary to the results of Jang et al. (2016) that green had the highest preference for indoor foliage plant leaf color for green interior, followed by patterned, red, purple, yellow, orange, and gray. It was also inconsistent with Ryou (2015) that green was the most preferred color of indoor plants planted in a workspace, and with Ou et al. (2004) that color is correlated to emo- tions and green particularly showed high preference. These conflicting results may be due to the fact that, since firefighters are always followed by danger due to the nature of their job, they are in an organization similar to the military, making them feel as if they are in a military life at work, thereby not preferring green that is most similar to the color of military uniform. The fact that red is not preferred seems related to the wounds and blood they see in accidents. Therefore, since most indoor plants are green, it is necessary to choose various colors of indoor foliage plants or use yellow with high preference mainly as the accent color while not using much red with low preference. However, since not enough research is conducted on plant 
Table 17. Correlation between preference of garden plants and emotional words for plant colors

\begin{tabular}{lllllll}
\hline & White & Green & Red & Yellow & Orange & Blue \\
\hline Dark-bright & .169 & .179 & .157 & $.208^{*}$ & .157 & .165 \\
Murky-clean & .125 & .192 & $.250^{*}$ & $.280^{* *}$ & .192 & $.195^{*}$ \\
Heavy-light & $.219^{*}$ & .145 & $.239^{*}$ & .161 & $.294^{* *}$ & $.204^{*}$ \\
Cool-warm & $.199^{*}$ & .031 & $.236^{*}$ & $.322^{* * *}$ & $.346^{* * *}$ & .171 \\
Stuffy-fresh & .190 & .185 & .116 & .084 & $.264^{* *}$ & $.279^{* *}$ \\
Strong-pale & .175 & .130 & .071 & .084 & $.253^{* *}$ & $.296^{* *}$ \\
Distracted-concentrated & $.199^{*}$ & .189 & .162 & $.258^{* *}$ & $.344^{* * *}$ & $.362^{* * *}$ \\
Depressed-spirited & .191 & .078 & $.259^{* *}$ & .146 & $.258^{* *}$ & $.304^{* *}$ \\
Nervous-relaxed & .192 & .097 & $.247^{*}$ & .176 & $.234^{*}$ & $.260^{* *}$ \\
Dislike-like & $.216^{*}$ & $.226^{*}$ & $.217^{*}$ & .176 & $.380^{* * *}$ & $.397^{* * *}$ \\
Vibrant-calm & $.251^{* *}$ & $.230^{*}$ & .133 & $.203^{*}$ & $.263^{* *}$ & $.410^{* * *}$ \\
Uneasy-comfortable & .185 & .171 & .188 & $.205^{*}$ & $.288^{* *}$ & $.338^{* * *}$ \\
Modes-Fancy & .148 & .104 & .144 & .138 & $.247^{*}$ & .137 \\
\hline
\end{tabular}

${ }^{*} p<.05, \stackrel{* *}{p}<.01,{ }^{* * *} p<.001$.

Table 18. Correlation between preference of vegetables plants and emotional words for plant colors

\begin{tabular}{|c|c|c|c|c|c|c|}
\hline & White & Green & Red & Yellow & Orange & Blue \\
\hline Dark-bright & $.202^{*}$ & $.266^{* *}$ & $.241^{*}$ & $.212^{*}$ & .096 & .193 \\
\hline Murky-clean & .151 & $.217^{*}$ & $.242^{*}$ & $.294^{* *}$ & .100 & $.221^{*}$ \\
\hline Heavy-light & .150 & .125 & .166 & $.207^{*}$ & $.221^{*}$ & .179 \\
\hline Cool-warm & $.215^{*}$ & .003 & .159 & $.208^{*}$ & .163 & .141 \\
\hline Stuffy-fresh & .103 & $.197^{*}$ & .135 & .122 & .119 & .054 \\
\hline Strong-pale & .133 & .068 & -.054 & -.005 & .079 & .125 \\
\hline Distracted-concentrated & $.202^{*}$ & .072 & $.198^{*}$ & $.241^{*}$ & .112 & $.244^{*}$ \\
\hline Depressed-spirited & .151 & -.001 & .153 & .170 & .085 & .121 \\
\hline Nervous-relaxed & .140 & .036 & $.213^{*}$ & .168 & .087 & .145 \\
\hline Dislike-like & .138 & $.199^{*}$ & .172 & $.256^{* *}$ & .192 & $.238^{*}$ \\
\hline Vibrant-calm & .162 & .185 & .131 & .130 & .133 & $.269^{* *}$ \\
\hline Uneasy-comfortable & .137 & $.212^{*}$ & .109 & .168 & .083 & .169 \\
\hline Modes-Fancy & $.317^{* * *}$ & .082 & .175 & $.281^{* *}$ & .075 & .067 \\
\hline
\end{tabular}

${ }^{*} p<.05,{ }^{* *} p<.01$.

color preference of firefighters showing conflicting results to the preference of non-firefighters, there is a need to conduct continuous research for more evidence.

\section{Correlation between plant preference and plant color emotion words of firefighters}

As a result of examining the correlation between flowering and vegetable plant preference and plant color emotion words, there was mostly a positive correlation between plant preference and plant color emotion words. The results of examining the correlation between flowering plant preference and plant color emotion words showed that there was highest positive correlation between white, green and 'dislike - like', 'vibrant - calm', red and 'depressed - spirited', 'murky - clean', 'nervous - relaxed', yellow and 'cool warm', 'murky - clean', 'distracted - concentrated', and orange and 'dislike - like', and there was positive correlation 
with 11 pairs of emotion words excluding 'dark - bright' and 'murky - clean'. Moreover, there was highest positive correlation between blue and 'vibrant - calm', and there was positive correlation with 10 pairs of emotion words (Table 17). The results of examining the correlation between vegetable plant preference and plant color emotion words showed that there was positive correlation between white and 'modest - fancy', 'distracted - concentrated', green and 'dark - bright', 'uneasy - comfortable', red and 'murky - clean', 'nervous - relaxed', yellow and 'modest - fancy', 'murky - clean', 'dislike - like', orange and 'heavy- light', and blue and 'distracted - concentrated', 'vibrant - calm', 'dislike - like', 'murky - clean' (Table 18). Higher preference in flowering and vegetable plants led mostly to more positive emotion words such as 'like', 'calm', 'spirited', 'relaxed', and 'concentrated', whereas lower preference led to more negative emotions such as 'dislike', 'vibrant', and 'depressed'. The result of this study that higher preference in flowering and vegetable plants can lead to higher emotions of 'like', 'calm', and 'relaxed' is supported by Jang et al. (2014) who analyzed the psychological effect of green office and discovered that greater green interior effect led to a positive correlation such as comfort and calmness.

\section{Conclusion}

This study surveyed and analyzed 117 male and female participants with the average age of $39.01 \pm 9.17$ to examine the plant type and color preference and conduct psychological assessment for gardening activities of firefighters in a high-risk occupational group. As a result of surveying plant experience and awareness of firefighters, among the three factors (plant growing experience, plant preference tendency, and plant-related event), plant-related event showed the highest score, followed by plant preference tendency and plant growing experience. The mean of the level of plant gardening activities was higher among older age and higher job position. We examined the quality of life according to the demographic characteristics of firefighters in a high-risk occupational group using the WHOQOLBREF (World Health Organization Quality of Life Assessment Instrument), and the result showed that the quality of life was higher among the $20 \mathrm{~s}-30 \mathrm{~s}$ than $50 \mathrm{~s}$, firefighter position than fire lieutenant or higher, those with higher average monthly income, and the singles group than married $(p<.05)$. Moreover, the administration group showed lower scores than the fire suppression group working in the field, showing statistical significance. Most firefighters showed high preference for herbal plants among the 12 types of flowering plants, in the order of Rosmarinus officinalis > Lavandula spp. > Mentha piperita, Calendula officinalis. Pelargonium spp. showed the least preference among the flowering plants. Among the 12 types of vegetable plants, fruit vegetable Lycopersicon esculentum showed the highest preference, and Angelica acutiloba the lowest preference with statistical significance. As a result of examining the preference in 6 plant colors, yellow was the most preferred color, followed by white, blue, orange, red, and green, showing statistical significance. We analyzed the responses of fire officials regarding the emotion words they had toward 6 plant colors, and discovered that 'bright' was the emotion felt most strongly by the white, yellow, and orange groups, 'calm' and 'comfortable' by the green and blue groups, and 'fancy' by the red group. Moreover, as for the correlation between flowering plant preference and plant color emotion words, there was a positive correlation between white, green and 'vibrant - calm', red and 'depressed spirited', yellow, orange and 'cool - warm', and blue and 'vibrant - calm'. As for the correlation between vegetable plant preference and plant color emotion words, there was a positive correlation between green and 'dark - bright', yellow and 'murky - clean', and blue and 'vibrant - calm'. Therefore, the results of this study suggest that the effect of agro-healing programs can be increased by using mostly yellow plants when creating indoor healing gardens at the workplaces of fire officials in a high-risk occupational group, and using herbal plants and Lycopersicon esculentum in the gardens for agro-healing activities. Moreover, higher preference of firefighters for flowering and vegetable plants may lead to more positive emotions such as 'like', 'calm' and 'relaxed'. Greater effect of green interior increases comfort and calmness, and higher attention restoration or positive emotions from the fascination of indoor/outdoor healing gardens and more memories related to plants lead to greater satisfaction and loyalty of visitors 
in the healing gardens. Creating indoor healing gardens with plants in the workplaces that are accessible to firefighters in a high-risk occupational group will provide them with emotional stability and peacefulness. Recently, the importance of firefighters is increasing significantly for disaster rescue and emergency medical services due to urbanization, but they are suffering more burden due to the increasing role and responsibility to meet the government needs for national safety. However, there is not much improvement in policies to protect physical and psychological stability of firefighters in reality. Therefore, increasing resilience of firefighters with plant growing experience or natural environment will improve the quality of life and relieve stress. The results of this study can be used in creating an indoor healing garden at workplaces of firefighters and applying plant types and colors to agro-healing activities.

\section{References}

Bak, B.G., S.Y. Lee, and J.H. Song. 2010. Development of ego-resiliency enhancement program and testing its effects. Korean J. Educ. Psychol. 24(1):61-82.

Chang, C.Y. and P.K. Chen. 2005. Human response to window views and indoor plants in the workplace. HortScience 40(5):1354-1359. https://doi.org/10.21273/HORTSCI.4 0.5 .1354

Choi, E.S. 2001. A model for post-traumatic stress and burnout in firefighters. J Korean Soc. Emerg. Med. Serv. 5(1):147-64.

Chong, W.S., C.U. Hong, and N.G. Kim. 2004. A study on human response to color light stimulation. Korean J. Sci. Emot. Sensib. 7(4):51-56.

Guilford, J.P. and P.C. Smith. 1959. A system of colorpreferences. Am. J. Psychol. 72(4):487-502. https://doi.org/ $10.2307 / 1419491$

Im, S.B. 2009. Theories in landscape analysis. Seoul, Korea: Seoul National University Press.

Jang, H.S., E.H. Yoo, K.J. Kim, and H.H. Jung, 2015. Preference and image perception for color and shape in green interior. J. People Plants Environ. 18(5): 413-420. https://doi.org/10.11628/ksppe.2015.18.5.413

Jang, H.S., G.M. Gim, S.J. Jeong, and J.S. Kim. 2019.
Assessment of display and events of agro-healing experience center for visitors satisfaction and revisit intention: A case study of 2018 Changwon agricultural technology exhibition. J. People Plants Environ. 22(1): 15-29. https://doi.org/10.11628/ksppe.2019.22.1.015

Jang, H.S., J.Y. Kim, K.S. Kim, and C.H. Pak. 2014. Human brain activity and emotional responses to plant color stimuli. Color Res. Appl. 39(3):307-316. https://doi.org/ $10.1002 / \mathrm{col} .21788$

Jang, H.S., K.J. Kim, E. Yoo, and H.H. Jung. 2016. Psychological effect of user for the color of cut- flower decoration in the conference place. J. People Plants Environ. 19(3):199-207. https://doi.org/10.11628/ksppe. 2016.19.3.199

Jang, H.S., S.W. Kang, and C.H. Pak. 2011. Influences of psychological effect and importance perception from the visual image of the indoor plants upon the repurchasing intention. J. Korean Soc. People Plants Environ. 14(2):121-131.

Janssens, J. 2001. Facade coulours not just a matter of personal taste - A psychological account of preferences for exterior building colours. Nord. J. Archit. Res. 34:17-21.

Kang, S.T. 2012. The relationship between the restriction on leisure, lifestyle and the quality of life on a police and a fire-fighting officer who is participated in leisure activity. Doctoral dissertation, Yongin University, Yongin, Korea.

Kaplan, R. and S. Kaplan. 1989. The Experience of nature: A psychological perspective. New York, NY: Cambridge University Press.

Kim, J.H. 2003. Studies on the tendency of Korean interior landscape and its application to architectural spaces. Master's thesis, Mokpo National University, Mokpo, Korea.

Kim, J.M., B.S. Suh, K.Y. Jung, D.I. Kim, W.S. Kim, H.S. Cho, J.W. Kim, J. Kwon, D.Y. Yoon, J.I. Kim, and Y.M. Roh. 2007. The study for musculoskeletal symptoms and job stress in firemen. J. Korean Soc. Occup. Environ. Hyg. 17(2):111-119.

Kim, Y.C., H.K. Chung, and S.H. Lee. 1989. A study on the life stress effects on psychiatric symptoms and physical condition in normal subjects. J. Korean Neuropsychiatr. Assoc. 28(2):282-291.

Ko, J.H. and K.R. Yun. 2007. The relationship between 
daily life stress and suicidal ideation of adolescents: The role of ego-resiliency as a buffer. Stud. Korean Youth 18(1):185-212.

Ko, Y.B. 2007. The influence factors of secondary traumatic stress of child protective service workers. Master's thesis. Ewha Woman's University, Seoul, Korea.

Lee, J.S. and K.C. Son. 1999. Effects of indoor plant and various colors stimuli on the changes of brain activity and emotional responses. J. Korean Soc. Hortic. Sci. 40(6):772-776.

Lee, N.H. and K.J. Bang. 1996. The influence of the ratio of greenery on the visual preference in interior landscape. J. Korean Inst. Landsc. Archit. 24(2):13-24.

Lee, S.H. 2019. A study on the effects of PTSD and quality of life among crime scene investigators: With a focus on the moderating effects of resilience. Master's thesis, Ajou University, Suwon, Korea.

Lee, S.H. and Y.H. Choi. 2006. A study on job stress and job satisfaction of 119 relief squads. J. Korean Acad. Community Health Nurs. 17(4):521-529.

Lee, S.Y. 2016. A study on the effects of PTSD and quality of life among firefighters -With a focus on the moderating effects of resilience-. Master's thesis, Pusan National University, Busan, Korea.

Lee, Y.J., H.Y. Min, and Y.M. Lee. 2004. The relationships between ego-resilience, adult attachment types, and college adjustment. Asian J. Educ. 5(4):125-144.

Min, S.K., C.I. Lee, K.I. Kim, S.Y. Suh, and D.K. Kim. 2000. Development of Korean version of WHO quality of life scale abbreviated version (WHOQOL-BREF). J. Korean Neuropsychiatr. Assoc. 39(3):571-579.

Oh, J.H. 2006. Analysis on influence factors of secondary traumatic stress, burnout, and physical symptpms for firefighters. Doctoral dissertation, Hanyang University, Seoul, Korea.

Osgood, C.E. 1952. The nature and measurement of meaning. Psychol. Bull. 49(3):197-237. https://doi.org/10.1037/h0 055737

Ou, L.C., M.R. Luo, A. Woodcock, and A. Wright. 2004. A study of colour emotion and colour preference. Part I: Colour emotions for single colours. Color Res. Appl. 29(3):232-240. https://doi.org/10.1002/col.20010

Park, M.G. 2015. Qualitative phenomenological research to PTSD experience of police crime scene officers. Master's thesis, Kyungsung University, Busan, Korea.

Parsons, R., L.G. Tassinary, R.S. Ulrich, M.R. Hebl, and M. Grossman-Alexander. 1998. The view from the road: Implications for stress recovery and immunization. J. Environ. Psychol. 18(2):113-139. https://doi.org/10.100 6/jevp.1998.0086

Rural Development Administration. 2017. Technology dissemination manual of preventative oriented type agrohealing. Jeonju, Korea: Author.

Ryou, S.M. 2015. Analysis of the preference level of color and color scheme of indoor plants by workplace. Master's thesis, University of Seoul, Seoul, Korea.

Ryu, J., E. Ha, K. Jeong-Choi, J.E. Kim, S. Park, and H. Kim. 2017. Firefighters and posttraumatic stress disorder. Korean J. Biol. Psychiatry 24(1):10-18. https://doi.org/1 0.22857/kjbp.2017.24.1.002

Sisa IN. 2013, October 17. Social welfare officials 39\% 'High risk stress'. Retrieved from http://www.sisain.co.kr

Son, K.C., S.K. Park, H.O. Boo, K.Y. Paek, S.H. Lee, and B.K. Huh. 2003. Horticultural therapy. Seoul, Korea: Joongang Life Publishing Co.

Song, J.E. 2004. Effect of interior plantscape in office on psycho-physiological improvement and stress alleviation of indoor workers. Doctoral dissertation, Konkuk University, Seoul, Korea.

Ulrich, R.S., R.F. Simons, B.D. Losito, E. Fiorito, M.A. Milis, and M. Zelson. 1991. Stress recovery during exposure to natural and urban environments. J. Environ. Psychol. 11(3):201-230. https://doi.org/10.1016/S02724944(05)80184-7

Valdez, P. and A. Mehrabian. 1994. Effects of color on emotions. J. Exp. Psychol.: Gen. 123(4):394-409. https://doi.org/10.1037/0096-3445.123.4.394

Whang, M.C., E.K. Ryu, E.H. Beyn, and C.J. Kim. 1997. Normalized sensitivity using EEG as an objective emotional index. Proceedings of the Korean Society for Emotion \& Sensitivity Conference (pp. 80-84).

Whoqol Group. 1995. The world health organization quality of life assessment (WHOQOL): Position paper from the World Health Organization. Soc. Sci. Med. 41(10): 1403-1409. https://doi.org/10.1016/0277-9536(95)00112-K

Whoqol Group. 1998. The world health organization qual- 
ity of life assessment (WHOQOL): Development and general psychometric properties. Soc. Sci. Med. 46(12): 1569-1585. https://doi.org/10.1016/S0277-9536(98)00009-4

Yun, Y.J. and S.W. Hong. 2018. Effects of job stress and burnout on the quality of life among 119 EMTs, Korean J. Emerg. Med. Serv. 22(3):131-148. https://doi.org/10.1 4408/KJEMS.2018.22.3.131 